\title{
An Analytical Model of Multiarc Sprag Clutch considering Geometry and Internal Interaction during Engagement
}

\author{
Chuang Huang, Ming Liu, and Yongqiang Zhao \\ School of Mechatronics Engineering, Harbin Institute of Technology, Harbin, China \\ Correspondence should be addressed to Chuang Huang; hcyl0312@gmail.com
}

Received 30 November 2016; Accepted 19 February 2017; Published 21 March 2017

Academic Editor: Jussi Sopanen

Copyright (c) 2017 Chuang Huang et al. This is an open access article distributed under the Creative Commons Attribution License, which permits unrestricted use, distribution, and reproduction in any medium, provided the original work is properly cited.

\begin{abstract}
A new multiarc sprag clutch model considering geometry and internal interaction during engagement is proposed in this paper. To increase the accuracy of the model, an improved model of geometric deformation coordination is presented to describe the basic geometrical quantities after rotation of the sprag. Then, based on this model, a novel nonlinear iteration method focusing on the varied contact radius is proposed to compute the normal contact force and show a good agreement with the FEM model. In addition, the alternate friction model considering stationary and rate-dependency friction is formulated and applied in the contacts of both the inner race and outer race. The proposed model is verified by comparison with other published results and experimental results. In subsequent analysis, the new model fully reveals the dynamical behaviors of the multiarc clutch during preload engagement under torque excitation; therefore, it will be useful for the performance analysis and dynamic design of multiarc sprag clutch.
\end{abstract}

\section{Introduction}

The circular-arc sprag clutch is the type most commonly used today in applications such as the helicopter transmission and passing and cutting torque from the power source. To provide the greatest possible life and torque capacity, the sprag has attained a high degree of refinement with precisionmachined compound curves such as multiarc, which provide optimum contact angles for driving and permitting higher transmitted torques for allowable Hertz and hoop stresses. Therefore, the performance of the sprag clutch is directly affected by geometric parameters, especially the contact compound curve of the sprag. Lynwander et al. [1] presented the design procedure for multiarc sprag clutch from the static view, including the deformations of the components and the geometric relationship. Kish [2] further proposed the iterative design method for single-arc sprag clutch containing additional centrifugal deformation of the outer race. However, the dynamic behavior of the sprag clutch cannot be explained from the perspective of statics, and failure still occurs during operation for the excessive dynamic pressure on the axial ends of the sprags or the wear caused by stick-slip during engagement. To address these problematic dynamic behaviors, it is essential to thoroughly understand the mechanism of the sprag clutch and to recognize the factors that affect its performance.

Figure 1 shows a manufacturers cut-out view of a complete sprag clutch, while Figure 2 gives a simplified schematic of its basic components. Such a sprag clutch is composed of five components: accurately formed sprags, a cylindrical inner race, a cylindrical outer race, energizing springs, and a sprag cage. Each sprag is essentially a strut placed between the races in such a way that it transmits power from one race to the other by an engaging action when either race is rotated in the driving direction. Rotation in the other direction disengages the sprags and the clutch is free or overruns. Either race may be the driven member or driving member. Note that the energizing spring plays a key role in the transition between the engagement and overrunning, as the contact normal force is very small at this time. In most circumstance, the specific compound curves such as multiarc are applied on the inner contact profile of the sprag, while the single circular of the outer contact profile remains unchanged.

Because the sprag clutch is a type of friction component with interactions in components due to the engaging action, 


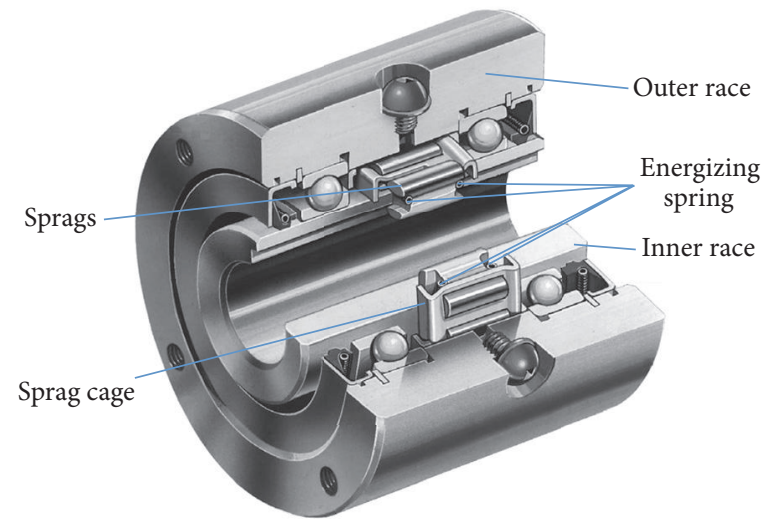

FIgURE 1: A cross-sectional view of Formsprag indexing clutch model.
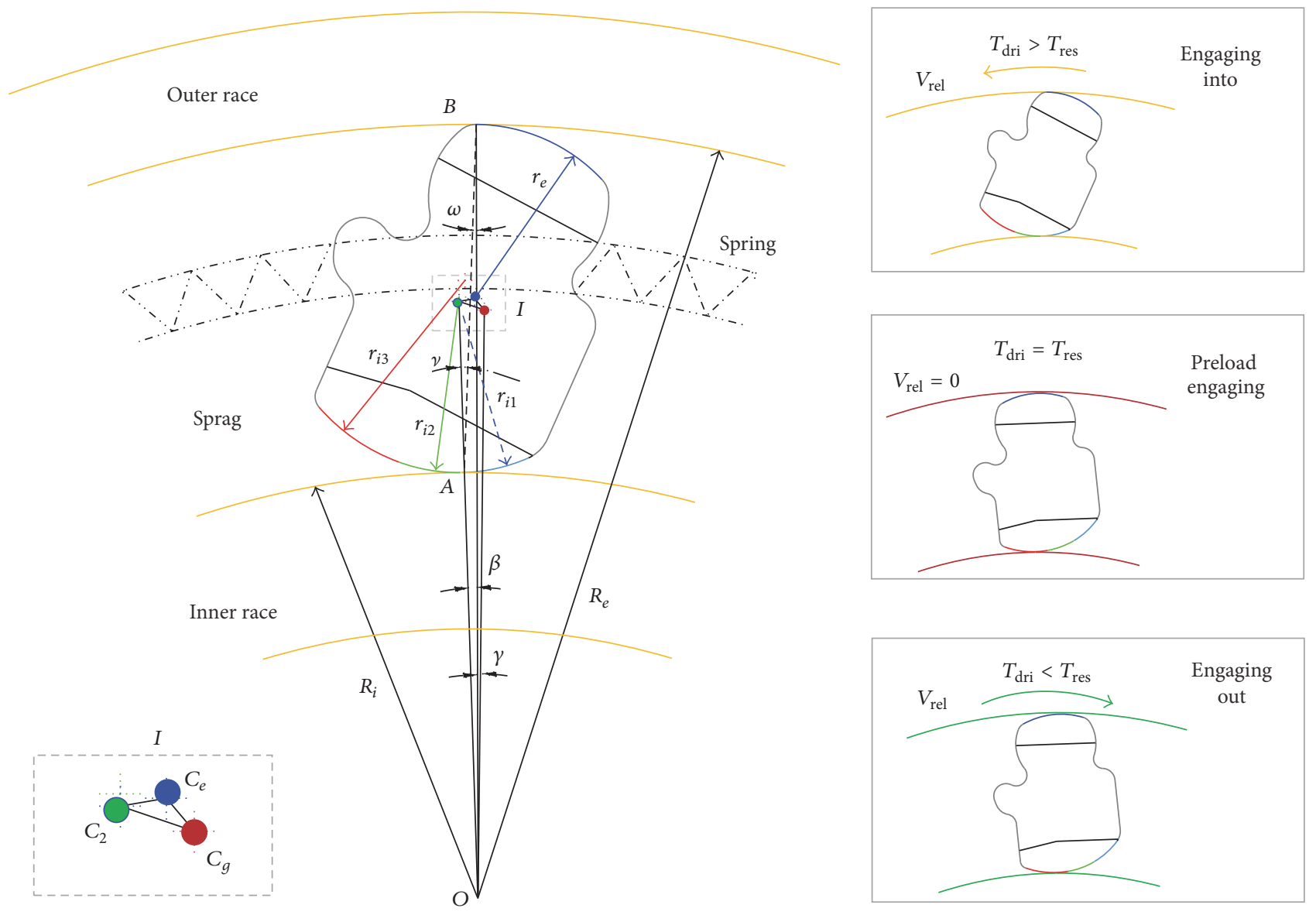

FIGURE 2: A schematic diagram of a multiarc sprag clutch and engaging conditions.

its geometric deformation coordination, contact nonlinearity, and friction are the main attributes responsible for its dynamic performance during engagement. These attributes have been studied extensively over the last twenty years.

Initially, total torsional stiffness and damping models are used to describe the dynamic characteristics of the singlearc sprag clutch, and then stepwise refined models have been proposed for single-arc sprag clutch concerning the geometry and internal interactions. The interactions include the normal contact forces and the tangential frictional forces between the sprags and races. Chassapis and Lowen [3] presented the torsional stiffness-damping model of a single-arc sprag clutch to simulate the dynamic behavior of a press-feed mechanism, in which energy dissipation was described by a 
linear damping model. Based on the work, Xu and Lowen [4] put forward the improved total torsional stiffness-damping model with a nonlinear torsional damping, which gave better agreement with experiment results. Further, $\mathrm{Xu}$ and Lowen [5] proposed a complete mathematical model, using an epicyclical train model to describe the geometry deformation coordination and motions of components and deriving the calculation method of the normal contact force, whereas it assumed pure rolling in contacts without slipping. Based on the same epicyclical train model, Liu et al. [6] presented a mathematical model of logarithmic spiral type sprag clutch for design and analysis, taking advantage of Hunt-Crossley contact impact theory to calculate the normal force but also neglected the slip in contacts. To investigate the dynamic behavior of stick-slip, Vernay et al. $[7,8]$ adapted a Coulomb friction model to describe the tangential interactions between the sprags and the inner race, and the structural deformations of the races were firstly considered in the geometry deformation coordination model; however, no slip was assumed in the contacts between the sprags and the outer race, and the edge contact was neglected. In addition, researchers constructed the model of single-arc sprag clutch on the software platform such as ADAMS $[9,10]$.

From the above discussions, to establish accurate dynamic modelling of the sprag clutch, three key issues should be discussed, including geometry deformation coordination model, the calculation method of the normal contact force, and the friction model in contacts. Compared to the simplified epicyclic train model only including local contact deformations, the geometry deformation coordination model developed by Vernay further took structural deformations into account. However, the centroid position of the sprag was assumed and the edge contact was not considered, which may lead to the axial end crushing on the sprag due to excessive contact stress. To the best of the author's knowledge, public literature mainly predicted about the dynamic performance of the single-arc sprag clutch. No studies have constructed a multiarc sprag model concerning the additional contact nonlinearity of the multiarc compound curve. Additionally, existing work on the friction model adopted the Coulomb friction model in the inner race contacts, which could reflect the behavior of stick-slip but did not consider the rate-dependency friction in boundary lubrication and the friction in the contacts of the outer race.

The purpose of this paper is to develop a multiarc sprag clutch model considering geometry and internal interaction, fully reflecting the dynamic behavior of multiarc sprag clutch during engagement. First, to improve the accuracy of the model, an improved geometric deformation coordination model is presented, considering the actual centroid position of the sprag, the non-Hertzian local contact deformations by the slice method, and structural deformation of the races. Second, to consider the contact nonlinearity of the multiarc compound curve, a novel nonlinear iteration method focusing on the varied contact radius is proposed to compute the normal contact force. Third, the alternate friction considering stationary and rate-dependency friction is formulated and applied in the contacts of both the inner race and the outer race, increasing a degree of revolution for the whole model of the sprag clutch.

This paper will be organized as follows. In the following section, the calculation method of the normal contact force and friction force in contacts are proposed, and a multiarc sprag clutch model considering geometry and internal interaction is established. In the validation section, the mathematical equations of the model are solved and the theoretical responses are compared to published results and experiment results. In the results and discussion section, the dynamic performance of multiarc sprag clutch is discussed during the preload engaging with torque excitation. In the last section, some conclusions are given.

\section{The Problem Formulation}

Figure 2 shows a simplified configuration of a complete sprag clutch in initial contact position and three sketch maps to illustrate the changes of engagement conditions. In initial contact position, uniform sprags are evenly distributed in annular space between the races and held in contact with the races by the energizing springs. The inner contact surface of the sprag is composed of three tangent circles with their own radius and center positions, and the switching of the circles in contact depends on the wedging position. The associated contact angles $\omega$ and $v$ are expressed with respect to a line connecting the inner and outer contact points $A$ and $B$, and $\beta$ and $\gamma$ are described by the lines $O C_{i}$ and $O C_{g}$ with respect to the line $O C_{e}$, respectively. Particularly, unlike the previous research, the centroid position of the sprag $C_{g}$ has no assumption on it and only depends on the mass distribution of the sprag. $R_{e}$ is the radius of inner surface of the outer race; $R_{i}$ is the radius of outer surface of the inner race; $r_{e}$ is the radius of outer surface of the sprag, and $C_{e}$ is the corresponding center position; the $r_{i 1}, r_{i 2}$, and $r_{i 3}$ are the different radius of inner surface of the sprag composed of three tangent circles, and $C_{i}$ is a general term for the center positions $C_{1}, C_{2}$, and $C_{3}$ changing with the wedging position.

As shown in Figure 2, there are three types of engagement conditions in the sprag clutch: Engaging into, preload engaging, and Engaging out. "Engaging into" sprags roll into a wedging position between the races when the driving torque on the outer race is sufficient to overcome the resisting torque on the inner race; otherwise, the "Engaging into" sprags become the "Engaging out" sprags until the sprags roll out of engagement. When "Engaging into" sprags stop the rotation with the driving torque, "Engaging into" sprags convert to "pre-load" engagement sprags, in which the races have no relative motion, and the driving torque is taken as the preload applied to the outer race.

A mode describing the real engagement process of the sprag clutch would be extremely complex. In order to obtain an effective and practical mathematical model, the following assumptions are made.

(1) All sprags are wedged simultaneously.

(2) Sprags are circumferentially uniform and share the load evenly. 


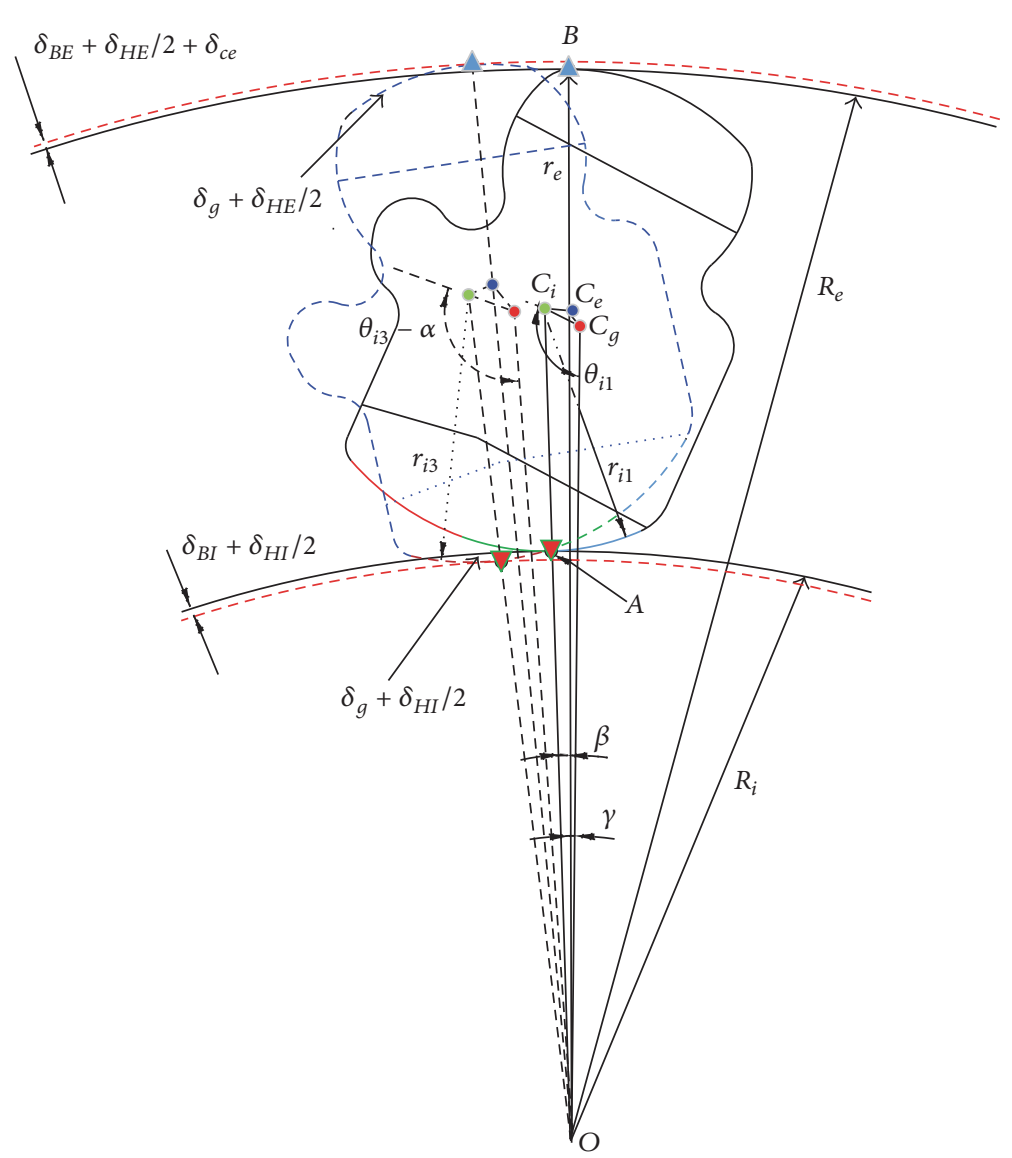

FIgURE 3: The deformations of components after rotation of the sprag.

(3) The inner race and the outer race are concentric definitely, without considering the manufacturing precision and the installation error of the sprag.

\subsection{The Geometry Deformation Coordination}

2.1.1. The Deformations in Components. In actual engagement of the sprag clutch, the sprags and the races deform with the rotation of the sprags, and substantial errors would occur if they are still considered as rigid components. To consider the deformations of components, the geometry deformation coordination model has been included in previous study, but the centroid position of the sprag was assumed to simplify the model and the local deformation did not consider edge contact. To improve the accuracy of the model, an improved model of geometry deformation coordination is proposed in this paper. The actual centroid position is included, and the edge contact is in consideration by the slice method, which has been effectively adapted in roller bearing [11].

Figure 3 shows the deformations of components after rotation of the sprag. Note that the sprags rotate and force the races to deform radially and compress the sprags from the orientation of contact points $A$ and $B$, when the sprag clutch is in "Engaging into" condition. As a result of the wedging action, two types of deformations are generated: the local contact deformation and the structural deformation. The local deformations of the sprags interacting the outer and inner race are defined as $\delta_{H E}$ and $\delta_{H I}$, respectively. The structural deformations for the inner and outer race are expressed as $\delta_{B I}$ and $\delta_{B E}$, and the structural deformation for sprags is written as $\delta_{g}$. Particularly, the additional centrifugal deformation of the outer race is defined as $\delta_{c e}$. The following discusses the calculation method used to obtain the deformations and basic geometric quantities of the sprag clutch after rotation.

The local contact deformations $\delta_{H E}$ and $\delta_{H I}$ are calculated by the slice method [12] shown in Figure 4, in which the sprag is axially divided into a certain number of slices with equal length, and the interaction among the deflections of slices is established by the influence coefficient method. The functional relationship between load and deflection on each slice can be obtained as follows:

$$
\begin{aligned}
& {\left[\mathbf{S}_{w}\right]_{i} \cdot\{\mathbf{q}\}_{i}=\{\boldsymbol{\Delta}\}_{i},} \\
& {\left[\mathbf{S}_{w}\right]_{e} \cdot\{\mathbf{q}\}_{e}=\{\Delta\}_{e} .}
\end{aligned}
$$




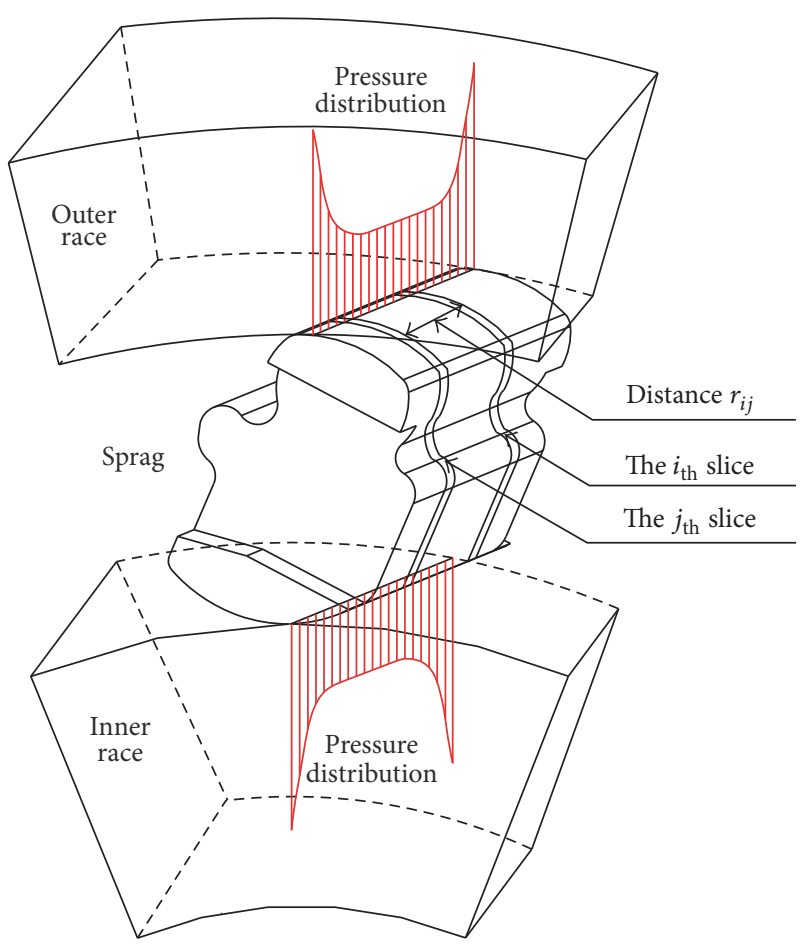

FIgURE 4: The slice method applied on contact between the sprag and the races.

In each of the above equations, $\left[\mathbf{S}_{w}\right]$ is the matrix of the weighted influence coefficients with specific expression:

$$
\begin{aligned}
& {\left[\mathbf{S}_{w}\right]=\frac{n}{\sum_{j, k} w_{j, k}}\left[\begin{array}{ccc}
s \cdot w_{j, k} & \cdots & s \cdot w_{j, n} \\
\vdots & \ddots & \vdots \\
s \cdot w_{n, k} & \cdots & s \cdot w_{n, n}
\end{array}\right]} \\
& \text { for } j=1 \cdots n, k=1 \cdots n \text {, }
\end{aligned}
$$

where $s$ are the elastic compliances associated with each slice in contact with either the inner or the outer race and $w_{j, k}$ is weighting function, meaning the influence of a unit pressure on $j$ slice on the deflection of the $k$ slice. Moreover, because the weighting function has the relationship $w_{j, k}=w_{k, j}$, therefore, $\left[\mathbf{S}_{w}\right]$ is a symmetric matrix.

$\{\mathbf{q}\}$ is the vector of unknown forces on each slice element of the sprag.

$$
\{\mathbf{q}\}=\left\{\begin{array}{c}
q_{j} \\
\vdots \\
q_{n}
\end{array}\right\} \quad \text { for } j=1 \cdots n .
$$

$\{\Delta\}$ is the vector of deflections belonging to each slice element of the sprag, and the deflection is used to represent the interpenetration between rigid contact partners.

$$
\{\Delta\}=\left\{\begin{array}{c}
\delta_{j}^{1 / e x} \\
\vdots \\
\delta_{n}^{1 / e x}
\end{array}\right\} \quad \text { for } j=1 \cdots n .
$$

The structural deformations for the races are calculated based on the Lame formula of thick-walled cylinder theory; the equations for structural deformation of the inner race $\delta_{B I}$ and that of the outer race $\delta_{B E}$ can be obtained as follows:

$$
\begin{aligned}
& \delta_{B E}=\frac{N_{b e} n}{2 \pi l E}\left[\frac{R_{e d}^{2}+R_{e}^{2}}{R_{e d}^{2}-R_{e}^{2}}+\nu\right], \\
& \delta_{B I}=\frac{N_{b i} n}{2 \pi l E}\left[\frac{R_{i}^{2}+R_{i d}^{2}}{R_{i}^{2}-R_{i d}^{2}}-v\right],
\end{aligned}
$$

where $l$ is the length of the sprag, $n$ is the number of the sprag, $R_{e d}$ is the radius of the outer surface of the outer race, $R_{i d}$ is the radius of the inner surface of the inner race, $E$ refer to the elastic modulus, $\nu$ represents Poisson's ratio, and $N_{b i}$ and $N_{b e}$ represent the normal contact force on the inner race or outer race, respectively.

With the structure deformation of the races, high radial loads are applied on both ends of the sprag and cause the deformation, which cannot be ignored. The compressive deflection for the sprag itself can be written as

$$
\delta_{g}=\frac{N_{\text {normal }}\left(R_{e}-R_{i}\right)}{b l E},
$$

where $N_{\text {normal }}$ is the normal contact force on the inner race or outer race. Either one for the calculation is reasonable, since their values are very close due to the small contact angles $\omega$ and $v$.

Moreover, the calculation of the additional centrifugal deformation for the outer race is based on the hollow disk theory. For high speed application, the outer race can be seen as a rotating disc with a central aperture due to its large radial dimension. Therefore, the additional deformation of the outer race can be written as

$$
\delta_{c e}=\frac{R_{e}}{E} \frac{(3+v) \rho}{4}\left(\frac{\pi n}{30}\right)^{2}\left[R_{e d}^{2}+R_{e}^{2}\left(\frac{1-v}{3+v}\right)\right] .
$$

2.1.2. Basic Geometric Quantities after Deformation. Based on the above deformations, the essential geometric quantities of length in the sprag clutch have corresponding changes and can be expressed as

$$
\begin{gathered}
O A=R_{i}-\delta_{B I}-\frac{\delta_{H I}}{2}, \\
O B=R_{e}+\delta_{B E}+\frac{\delta_{H E}}{2}+\delta_{c e}, \\
O C_{i}=R_{i}+r_{i}-\delta_{B I}-\frac{\delta_{g}}{2}-\delta_{H I}, \\
O C_{e}=R_{e}-r_{e}+\delta_{B E}+\frac{\delta_{g}}{2}+\delta_{H E} .
\end{gathered}
$$




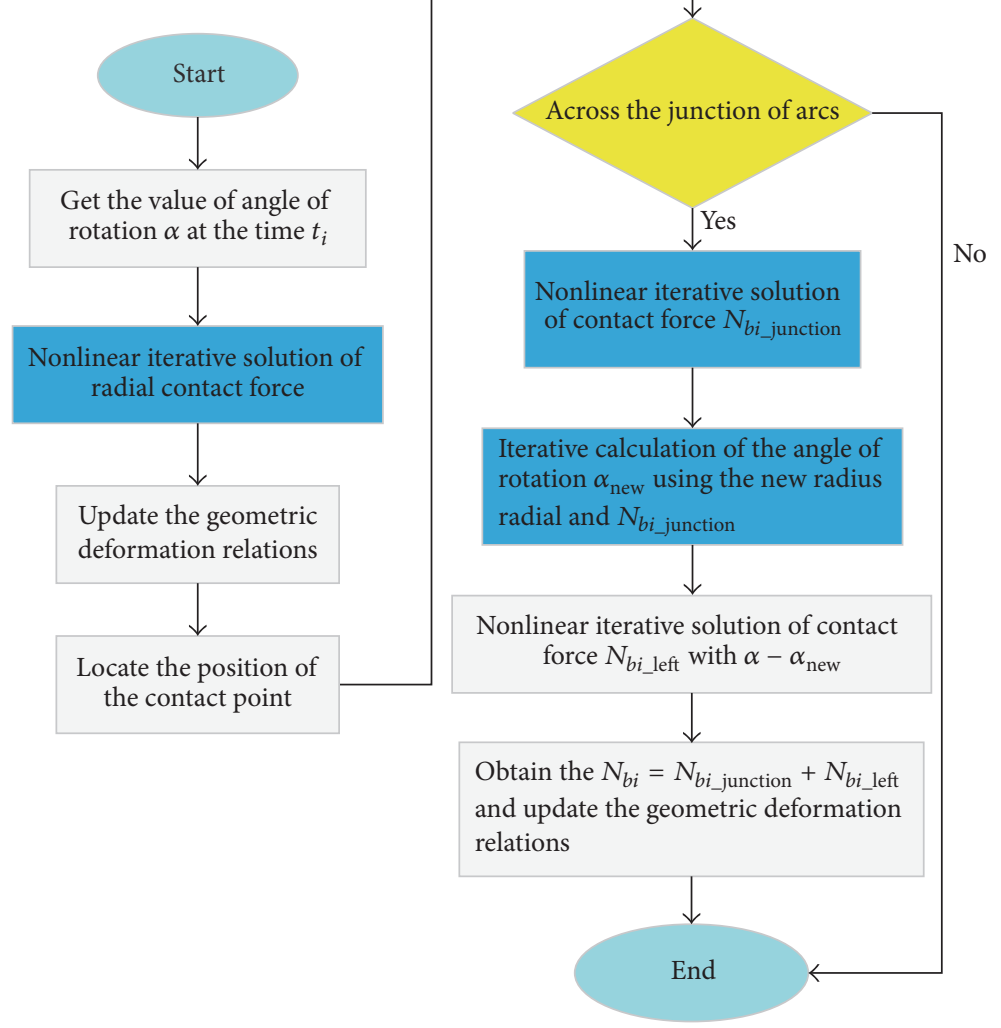

Figure 5: The flow chart of the proposed method of normal contact force.

As shown in Figure 3, the angles $\angle O C_{G} C_{i}, \beta$, and $\gamma$ vary with the changes of $O A, O B, O C_{i}$, and $O C_{e}$, and the equations of the angles can be expressed as

$$
\begin{aligned}
\angle O C_{G} C_{i} & =\theta_{i}-\alpha=a \cos \left(\frac{a^{2}+O C_{g}^{2}-O C_{i}^{2}}{2 \times C_{i} C_{g} \times O C_{G}}\right), \\
\beta & =a \cos \left(\frac{O C_{i}^{2}+O C_{e}^{2}-C_{i} C_{e}^{2}}{2 \times O C_{i} \times O C_{e}}\right), \\
\gamma & =a \cos \left(\frac{O C_{g}^{2}+O C_{e}^{2}-C_{g} C_{e}^{2}}{2 \times O C_{g} \times O C_{e}}\right),
\end{aligned}
$$

where $\theta_{i}$ is the initial value of the angle $\angle O C_{G} C_{i}$ in initial contact position and $\alpha$ is the rotation angle of the sprag.

2.1.3. Calculation of the Normal Contact Force. Based on the above equations of geometric deformation coordination, the normal contact force on the inner race would be obtained in each position and can be expressed as

$$
\begin{aligned}
& N_{b i} \\
& = \begin{cases}f\left(\alpha, R_{i}, R_{e}, r_{i}, r_{e}, O C_{i}, \delta_{H E}, \delta_{H I}, \delta_{B E}, \delta_{B I}, \delta_{g}, \delta_{c e}\right), & \alpha>0, \\
0, & \text { else, }\end{cases}
\end{aligned}
$$

where $r_{i}$ is a general term for $r_{i 1}, r_{i 2}$, and $r_{i 3}$, and $O C_{i}$ is a general term for $O C_{1}, O C_{2}$, and $O C_{3}$. Their values depend on the position of the contact point $A$ and change to the corresponding value when sprags roll across the junction point of tangent circles. Focusing on the varied contact radius on inner surface of the sprag, a novel nonlinear iteration method should be proposed to compute the normal contact force during whole engagement.

Figure 5 shows the mechanics of the novel nonlinear iteration method. First, a judgment is always carried out within each time step to determine whether the contact point has crossed the near junction point of tangent circles. If not, the calculation of normal contact force continues to proceed. Second, the normal contact force $N_{b i \text { junction }}$ is calculated when sprags rotate to the junction point, and the new rotation angle $\alpha_{\text {new }}$ is obtained using the new radius, corresponding center, and the normal contact force $N_{b i \text { junction }}$. Third, the additional normal contact force $N_{b i \text { left }}$ is given corresponding to the left rotation angle $\alpha-\alpha_{\text {new }}$ in the time step. At last, the total normal contact force is gained in the time step, passing through the junction point.

In order to verify the effectiveness of the proposed method, the torsional stiffness of multiarc sprag clutch is calculated to compare with the other two methods, using the measured parameters of sprag clutch [13]. As shown in the appendix, the calculated torsional stiffness by the slice method has a better agreement with the finite element method (FEM) results than the model based on Hertzian theory, reflecting the discontinuity of its change rate at the junction points of circles. 
2.2. Calculation of Friction Force. According to Vernay et al's research $[7,8]$, the motions between the sprags and races are pure rolling with no relative motions in most work time, and the friction state of interaction in sprag clutch belongs to the static friction phase with no lubrication. But when the required friction force keeping the pure rolling motion exceeds the maximum static friction, slip will occur in contacts and the relative motions exist between the sprags and races; because of the slip, lubrication should be considered in this phase, and the changeable sliding rate should take into account the friction force model to reflect the Stribeck effect. Therefore, as shown in Figure 6, the friction model should include static friction, rate-dependent friction, and the transformation between them $[14,15]$. To overcome the discontinuity of the transition point, a narrow band of zero velocity [16] is introduced. Taking the friction in the inner race for example, the expression of the alternate friction force can be written as

$$
T_{b i}\left(x, r_{i}\right)= \begin{cases}T_{b i f}\left(x, r_{i}\right), & \text { if }\left|\operatorname{Vel}_{b i}\right|<D_{V},\left|T_{b i f}\left(x, r_{i}\right)\right|<T_{b i s} \text { (stick) } \\ T_{b i s} \operatorname{sgn}\left(T_{b i f}\left(x, r_{i}\right)\right), & \text { if }\left|\operatorname{Vel}_{b i}\right|<D_{V},\left|T_{b i f}\left(x, r_{i}\right)\right|>T_{b i s} \text { (stick to slip transition) } \\ N_{b i} \mu_{b d}\left(\operatorname{Vel}_{b i}\right) \operatorname{sgn}\left(\operatorname{Vel}_{b i}\right), & \text { if }\left|\operatorname{Vel}_{b i}\right|>D_{V} \text { (slip) }\end{cases}
$$

with

$$
\mu_{b d}\left(\mathrm{Vel}_{b i}\right)=\mu_{c b}+\left(\mu_{s b}-\mu_{c b}\right) e^{-\gamma_{b}\left|\mathrm{Vel}_{b i}\right|},
$$

where $\mathrm{Vel}_{b i}$ is the sliding velocity at the contact point of inner race and $D_{V}$ is a narrow band of zero velocity. $x$ is the system state vector, $T_{b i f}\left(x, r_{i}\right)$ is the static force to keep the pure rolling contact, $T_{b i s}$ is the maximum static friction force, and $\mu_{b d}$ is the velocity-depending friction coefficient under boundary lubrication, in which the exponential decaying behavior is consistent with the experimental results [17].

Similar friction model is used to calculate the friction force on the outer race $T_{b e}$, and specific processes are followed to calculate the friction forces on the inner and outer races. First, the friction forces $T_{b i}$ and $T_{b e}$ are calculated to keep pure rolling at contact points, and $T_{b i}$ is always larger than $T_{b e}$ during engagement. Then, $\left|T_{b i f}\left(x, r_{i}\right)\right|$ is compared to the maximum static friction force $T_{b i s}$ in each time step. It must have $\left|T_{b i f}\left(x, r_{i}\right)\right|<T_{b i s}$ for the engagement without slip; otherwise, slip begins. The direction of the slip depends on the relative velocity at contact point. Additionally, the similar process is carried out to calculate the friction force on the outer race $\left|T_{b e f}\left(x, r_{e}\right)\right|$.

2.3. Differential Equations of Motion. Based on Newton's second law, the differential equations of the motion can be derived and describe the dynamic behavior of multiarc sprag clutch during engagement. To derive the equations of motion, first of all, it is necessary to analyze the forces acting on the sprag, the inner race, and the outer race.

The forces acting on the sprag are shown in Figure 7. $N_{b i}$ and $N_{b e}$ are the contact normal forces acting between the sprags and the races, $T_{b i}$ and $T_{b e}$ are the friction forces between the sprags and the races, $C_{r l}$ is the viscous damping between the races, $C_{b i}$ and $C_{b e}$ are the constant torques applied on the inner and outer races, respectively, $K_{r}$ is the stiffness of the elastic blades holding the sprags and the races in contact, $\alpha_{\max }$ is the assumed rotation angle of the sprag to ensure the initial contact torque formed by the energizing springs, and the $K_{r}$ and $\alpha_{\max }$ are calculated from the initial contact equilibrium equations.
The application of Newton's second law yields, for the sprags,

$$
\begin{aligned}
& I_{g}(\ddot{\alpha}+\ddot{\varphi})-b_{e} T_{b e}-K_{r}\left(\alpha_{\max }-\alpha\right)-b_{i} T_{b i}+h_{i} N_{b i} \\
& \quad-h_{e} N_{b e}=0, \\
& -m g O C_{g} \ddot{\varphi}-\cos (\beta+\gamma) T_{b i}+\sin (\beta+\gamma) N_{b i} \\
& \quad+T_{b e} \cos (\gamma)-N_{b e} \sin \gamma=0,
\end{aligned}
$$

with

$$
\begin{aligned}
& b_{i}=O C_{g} \cdot \cos (\beta+\gamma)-O A, \\
& b_{e}=O B-O C_{g} * \cos (\gamma), \\
& h_{i}=O C_{g} \cdot \sin (\beta+\gamma), \\
& h_{e}=O C_{g} \cdot \sin (\gamma),
\end{aligned}
$$

where $I_{g}$ is the inertia of the sprag, $I_{b i}$ and $I_{b e}$ are the inertias of the inner race and outer races, respectively, $\varphi$ and $\alpha$ are the two degrees of the sprag, $\varphi$ is the azimuthal angle of the azimuth coordinate system $\overrightarrow{o x_{G} y_{G}}$ with respect to the absolute coordinate system $\overrightarrow{o x_{o} y_{o}}$, and $\alpha$ is the rotational angle of the body fitted coordinate system $\overrightarrow{o x_{g} y_{g}}$ with respect to the azimuth coordinate system $\overrightarrow{o x_{G} y_{G}}$.

For the inner and outer race,

$$
\begin{aligned}
& I_{b i} \ddot{\theta}_{b i}-n_{s} T_{b i}\left(R_{i}-\delta_{B I}-\frac{\delta_{H I}}{2}\right)+C_{r l}\left(\dot{\theta}_{b i}-\dot{\theta}_{b e}\right) \\
& \quad-C_{b i}=0 \\
& I_{b e} \ddot{\theta}_{b e}+C_{r l}\left(\dot{\theta}_{b e}-\dot{\theta}_{b i}\right) \\
& \quad+n_{s} T_{b e}\left(R_{e}+\delta_{B E}+\frac{\delta_{H E}}{2}+\delta_{c e}\right)-C_{b e}=0
\end{aligned}
$$

where $\theta_{b i}, \theta_{b e}$ are the degrees of the inner and outer race, respectively. 


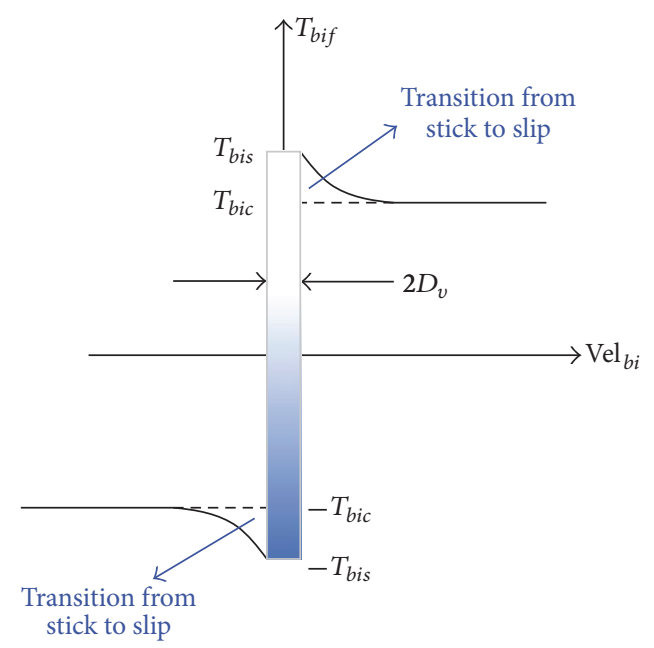

FIGURE 6: Alternate friction model considering static and rate-dependent friction.

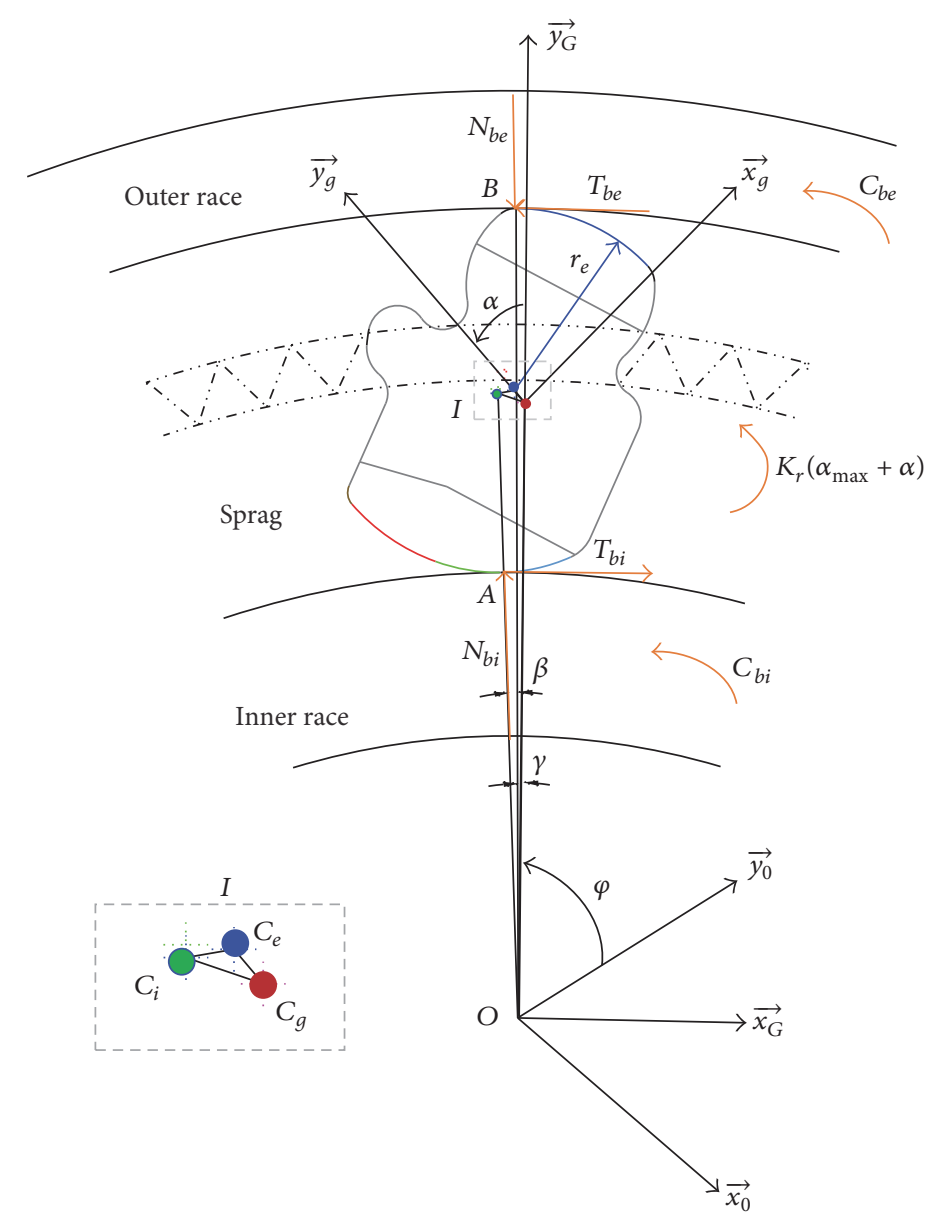

FIgURE 7: A schematic diagram of forces on the sprag.

The system equations of motion are nonlinear ordinary second-order differential equations. They can be solved using a fourth-order Runge-Kutta algorithm with fixed time step. The time step for the numerical procedure is chosen to be $\Delta_{t}=1 \times 10^{-5} \mathrm{~s}$.

\section{Validation}

To verify the effectiveness of the proposed model, the parameters and operating condition of Vernay's model are adapted, and a comparative analysis of the sprag clutch is carried out. 
TABLE 1: The parameters of the sprag clutch applied in the simulation.

\begin{tabular}{lc}
\hline Parameter & Value \\
\hline$n b g$ & 12 \\
$R_{i d}(\mathrm{~mm})$ & 7.21 \\
$R_{i}(\mathrm{~mm})$ & 11.11 \\
$R_{e}(\mathrm{~mm})$ & 19.44 \\
$R_{e d}(\mathrm{~mm})$ & 26.23 \\
$r_{i 1}(\mathrm{~mm})$ & 4.02 \\
$r_{i 2}(\mathrm{~mm})$ & 4.02 \\
$r_{i 3}(\mathrm{~mm})$ & 4.02 \\
$m_{g}(\mathrm{~kg})$ & $1.8 \times 10^{-3}$ \\
$I_{1}\left(\mathrm{~kg} \cdot \mathrm{m}^{2}\right)$ & $1.97 \times 10^{-4}$ \\
$I_{b i}\left(\mathrm{~kg} \cdot \mathrm{m}^{2}\right)$ & $1.41 \times 10^{-3}$ \\
$I_{b e}\left(\mathrm{~kg} \cdot \mathrm{m}^{2}\right)$ & $1.41 \times 10^{-3}$ \\
$I_{g}\left(\mathrm{~kg} \cdot \mathrm{m}^{2}\right)$ & $1.18 \times 10^{-8}$ \\
$K_{r}(\mathrm{Nm} / \mathrm{rad})$ & 0.01 \\
$C_{r l}(\mathrm{Nms} / \mathrm{rad})$ & 0.1 \\
$C_{f}(\mathrm{Nm})$ & $1.66 \times 10^{-2}$ \\
\hline
\end{tabular}

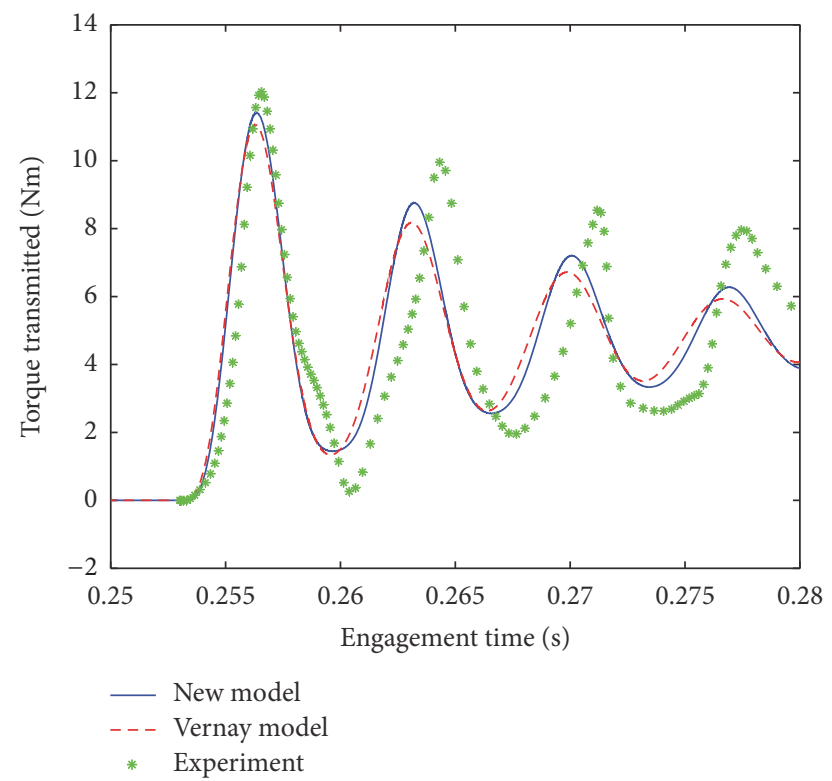

FIGURE 8: Comparison of results of the new model with results of Vernay's model and experiment.

By setting the same radius and center of the three circles, the multiarc sprag clutch became the single-arc sprag clutch shown in Table 1 . The operating condition is that firstly the inner race accelerates and moves to the free direction and then reverses direction and engages suddenly when the inner race meets the elastic obstacle on the torsion bench [8].

The comparison of the inner race speed process against the Vernay's model and the experimental data are shown in Figure 8. In general, the shape and amplitude of the curve obtained from the proposed model approximate the experiment results better than the curves from Vernay's model. This good agreement shows the effectiveness of the new model. Note that the damping in the simulation is considered constant equal to an average value obtained from Vernay's experiments, because the nonlinearity of stiffness is more important than the nonlinear damping on the dynamic behavior of the freewheel. This is the reason why the new model and the Vernay model agree with each other well and still have similar error profiles with respect to the experimental results. In addition, the friction forces are calculated using the alternate friction model to obtain the torque transmitted, focusing on the problem that whether slip exists in contacts during engagement. However, slip in initial engagement lasts only half thousandth of a second when the sprag clutch initially engages and has little effect on the torque transmitted $[7,8]$.

\section{Results and Discussion}

Based on the proposed model of multiarc sprag clutch, the dynamic behavior focusing on edge contact and stick-slip are investigated during preload engaging. To investigate the dynamic behavior, it is necessary to define the structural parameters and operating conditions for the sprag clutch. First, based on the parameters of single-circle sprag clutch listed in Table 1, A multiarc sprag clutch is formed by changing the suitable value of $r_{i 2}$ and $r_{i 3}$ to 4.08 and 4.15, respectively. Then, the centroid position coordinates are automatically obtained through the CAD model of the clutch using the macro programming method. Subsequently, the operating conditions are defined as follows: with the preload $C_{e 1}$ on the inner race, a sine torque excitation of $C_{e 2}=$ Amp $\times \sin (\Omega \times t)$ is applied to the outer race, and a high equivalent stiffness spring is assumed to connect the outer race to a fixed abutment [7]. Two different combinations for $C_{e 1}$ and $C_{e 2}$ are selected for preload engaging: one is the high torsional vibration excitation $C_{e 2}$ with respect to preload $C_{e 1}$, in which the amplitude of $C_{e 2}$ is assigned to $6 \mathrm{Nm}, 8 \mathrm{Nm}$, and $10 \mathrm{Nm}$, respectively, and $C_{e 1}$ is set to $3.34 \mathrm{Nm}$; the other is low torsional vibration excitation $C_{e 2}$ with respect to preload $C_{e 1}$, in which the amplitude of $C_{e 2}$ is assigned to $6 \mathrm{Nm}, 8 \mathrm{Nm}$, and $10 \mathrm{Nm}$ respectively, and $C_{e 1}$ is set to $16.8 \mathrm{Nm}$.

\subsection{Preload Engaging Suffering Torsional Vibration Excitation}

4.1.1. High Torsional Vibration Excitation with respect to Preload. Figure 9 shows abnormal disengagement phenomenon of multiarc sprag clutch, including the time varying of the rotation angle of the inner race, the contact stress distribution under different excitation torques, and the friction coefficient on the inner contact points.

The rotation angle of the inner race has a clear offset to the overrunning direction when $C_{e 2}$ is $8 \mathrm{Nm}$ and $10 \mathrm{Nm}$, but no offset when $C_{e 2}$ is $6 \mathrm{Nm}$. With the offset, the lower limit of the contact stress moves toward to zero, and the friction coefficient changes its value, transforming between the static and sliding friction coefficient. The reason for the offset is that slip appears in contacts, which moves the angle of the inner 


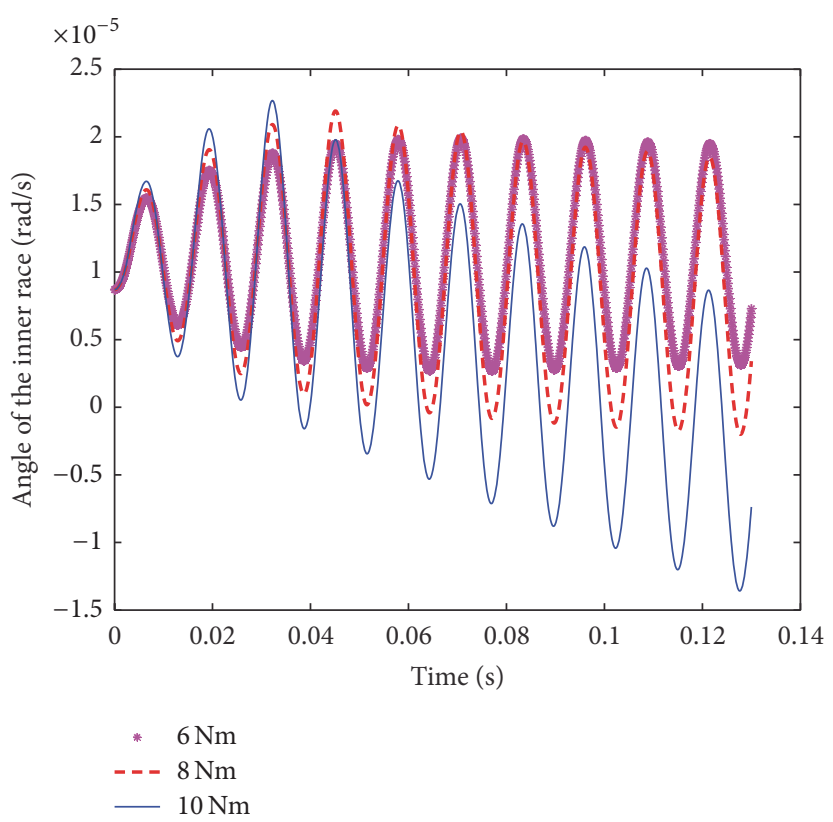

(a) Slip to the disengagement direction

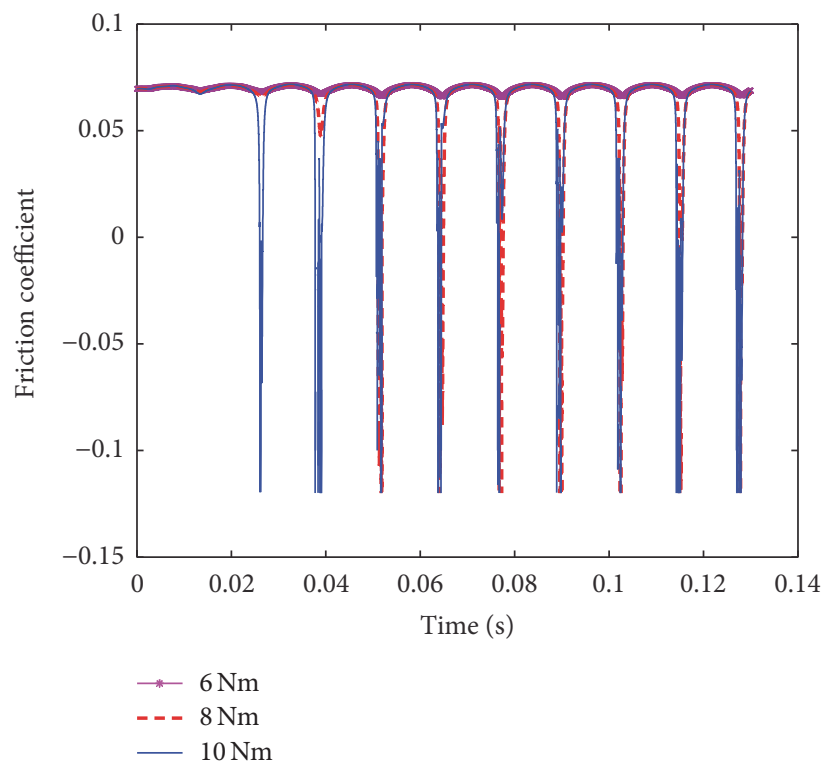

(c) Variation of friction coefficient

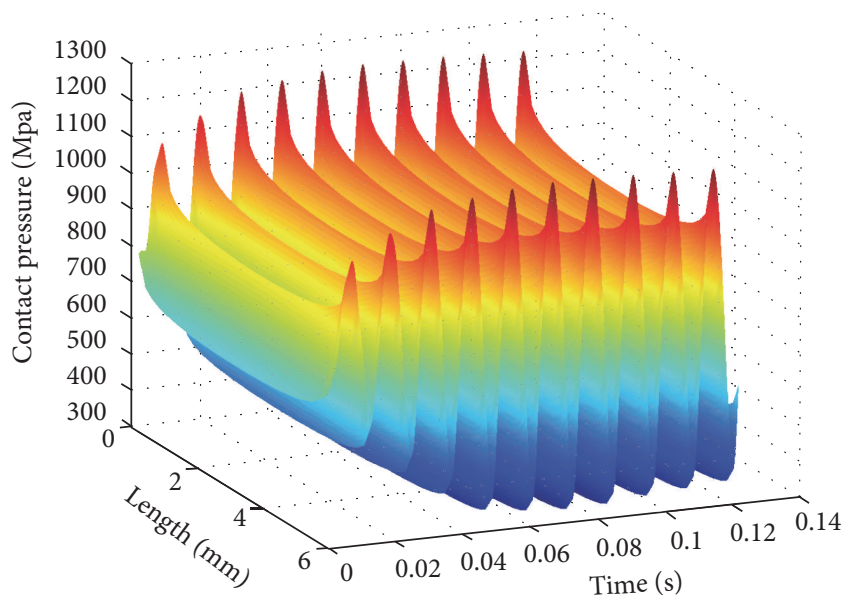

(b) Excitation torque $6 \mathrm{Nm}$

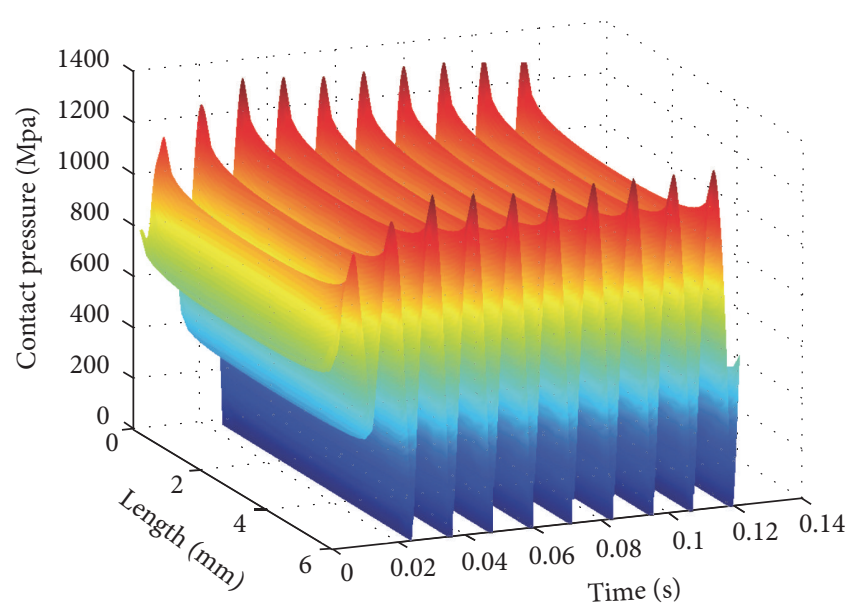

(d) Excitation torque $10 \mathrm{Nm}$

FIGURE 9: Abnormal disengagement phenomenon of multiarc sprag clutch.

race to the overrunning direction until out of the engagement. As shown in Figure 9(d), the contact stress is close to zero when the slip appears, indicating the sprags are about to depart from the races. At this time, the spring force exerted by the spring blade plays a key role to end the slip, holding the sprag to reengaging into the races, since the contact forces are small at this moment.

Note that the negative value of the friction coefficient means that the direction of the friction torque for the sprags is opposite to the engagement direction at the moment. As shown in Figure 9(c), the slip appears as the value of friction coefficient is more than the maximum static friction and the friction force changes its direction when the values of $C_{e 2}$ are $8 \mathrm{Nm}$ and $10 \mathrm{Nm}$. There is no slip when the value $C_{e 2}$ is $6 \mathrm{Nm}$, because the corresponding friction coefficient is no more than the maximum static friction.

4.1.2. Low Torsional Vibration Excitation with respect to Preload. According to Vernay's study, the maximum friction coefficient for steel-steel contact is dynamic and varies in a range of 0.04 to 0.12 during mixed lubrication. For this operating condition, slip does not occur when the maximum friction coefficient is near its upper limit but appears when the maximum friction coefficient is toward to its lower 


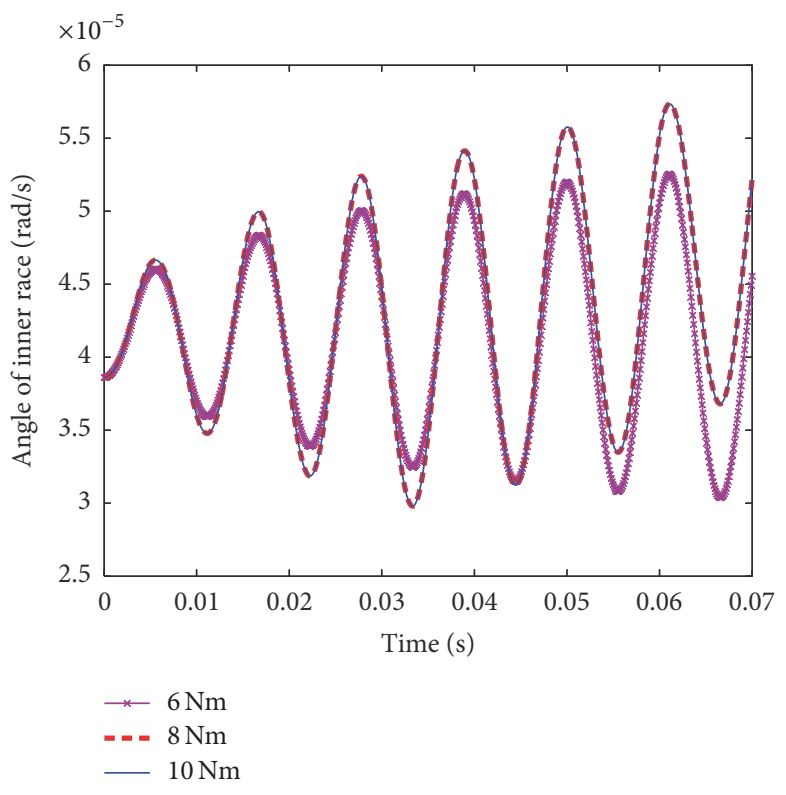

(a) Slip of angle of inner race to engagement direction

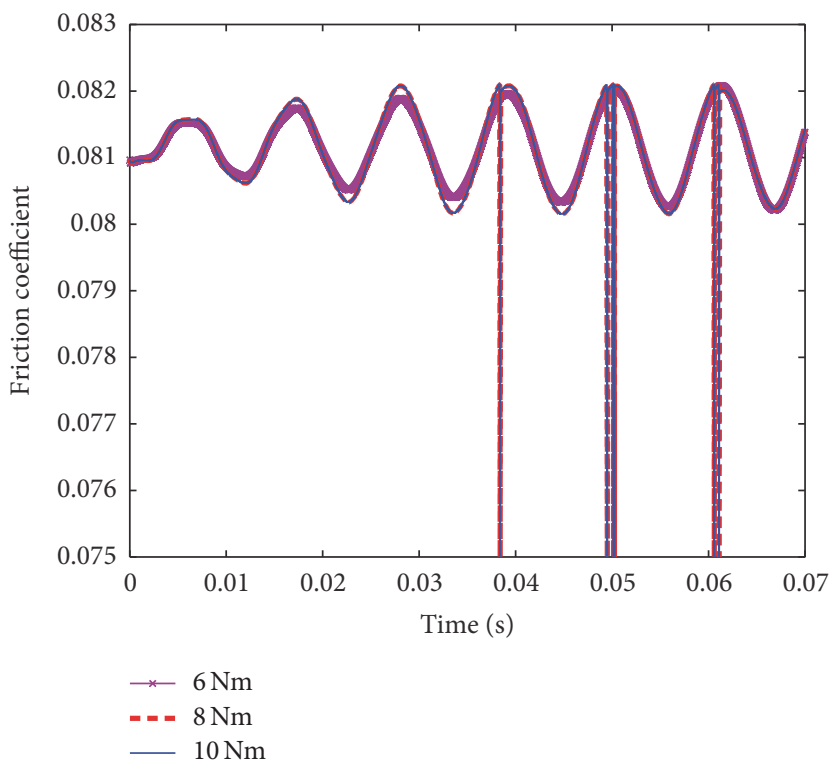

(c) Variation of friction coefficient

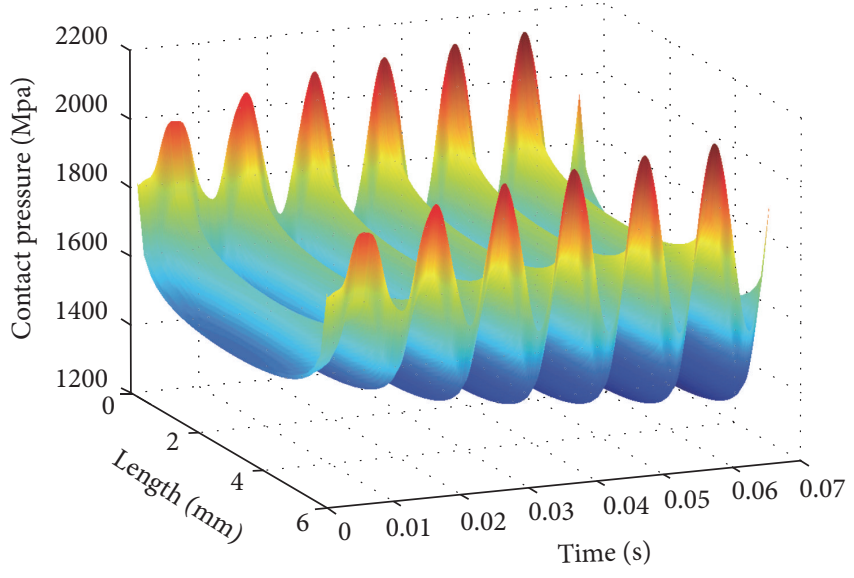

(b) Excitation torque $6 \mathrm{Nm}$

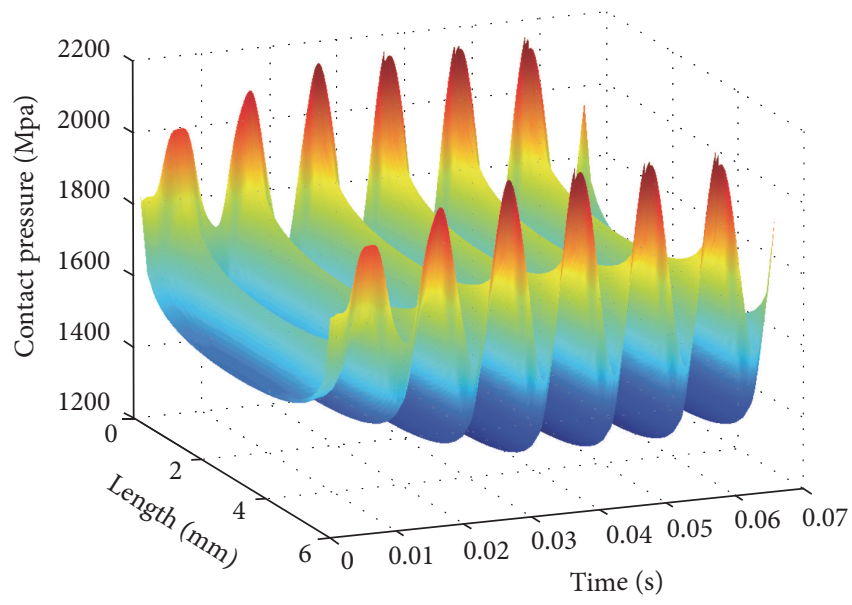

FIGURE 10: Slip phenomenon of multiarc sprag clutch under low torsional vibration excitation with respect to preload.

limit. As shown in Figure 10, slip occurs until the dynamic maximum friction coefficient is reduced to 0.082 . In addition, the loads on the races also influence the slip in contacts. When excitation torque $C_{e 2}$ is $8 \mathrm{Nm}$ and $10 \mathrm{Nm}$, the curves reveal the offset of the inner race moving to the direction of engagement, rather than the direction of overrunning, but no offset when the value $C_{e 2}$ is $6 \mathrm{Nm}$.

Note that the dynamic contact stress fluctuates around the reference static contact stress determined by the preload, while it would be of the same order of magnitude with allowable contact stress under rated preload torque. If slip occurs with high contact stress, serious wear can be caused on surface of the sprag. Therefore, the friction coefficient cannot be lower than a certain value in this operating condition, which puts forward requirements for surface material, surface technology, and lubrication design for the sprag clutch.

\section{Conclusion}

To understand the dynamic behavior of multiarc sprag clutch, a new multiarc sprag clutch is proposed to consider the geometry and interaction in this paper. The following conclusions can be drawn from the work presented in this paper. 
(1) To improve the accuracy of the model, an improved model of geometric deformation coordination considering centroid position and edge contact have been presented. Based on the improved model, the calculated torsional stiffness has better agreement with FEM results than the previous model, and the dynamic behavior of the sprag clutch reveals the contact stress concentration throughout the engagement.

(2) To reflect the contact nonlinearity of the multiarc compound curve, a novel nonlinear iteration method of normal contact force is proposed. Using the method, the calculated torsional stiffness of multiarc sprag clutch has good agreement with FEM results, and the change rate of it presents the discontinuity at the junction points of circles.

(3) The alternate friction model considering stationary and rate-dependency friction is formulated and applied in the contacts of both the inner race and outer race, increasing a degree of revolution for the whole model of the sprag clutch. Due to the slip in contacts, the sprags may disengage with the races in the condition of high torsional vibration excitation with respect to preload, and serious wear would be caused in the condition of low torsional vibration excitation with respect to preload, when the maximum friction coefficient is toward its lower limit.

(4) The proposed model of multiarc sprag clutch is effective to reflect the dynamic behavior during engagement, especially the edge contact and stick-slip. Using this model, further research can be performed to determine the key influencing factors and help to modify the design and control parameters of multiarc sprag clutch.

\section{Appendix}

The following is the comparison of the torsional stiffness of multiarc sprag clutch through three different calculation methods of the local deformation: Hertzian contact theory, the slice method, and FEM. First, the windup angle is obtained by the equations of the pure rolling in contact points, and then the torque transmitted is calculated through the static equilibrium equations of the sprag shown in Figure 7 . Then, the torsional stiffness is easily obtained by the division of them, and the curves are shown in Figure 12.

The pure rolling in contact points means that the relative sliding velocity of the contact partners is zero, so the equations for the pure rolling in contact point $A$ in Figure 7 are deduced as

$$
\begin{aligned}
& \underset{A \in B I / O}{\vec{V}} \cdot \overrightarrow{x_{A}}=\underset{A \in g / O}{\vec{V}} \cdot \overrightarrow{x_{A}}, \\
& \underset{A \in B I / O}{\vec{V}} \cdot \overrightarrow{x_{A}}=-\left(R_{i}-\delta_{B I}-\frac{\delta_{H I}}{2}\right) \times \dot{\theta}_{B I},
\end{aligned}
$$

$$
\begin{aligned}
\overrightarrow{A \in g / O}_{\vec{V}}= & \left(R_{e}-r_{e}+\delta_{B E}+\delta_{c e}+\frac{\delta_{g}}{2}+\delta_{H O}\right) \\
& \times \dot{\varphi} \times \overrightarrow{x_{B}}+b \times(\dot{\alpha}+\dot{\varphi}) \times \overrightarrow{x_{A}}+h \\
& \times(\dot{\alpha}+\dot{\varphi}) \times \overrightarrow{y_{A}}, \\
\dot{\theta}_{b i}= & \dot{\varphi}-\frac{b_{i}}{R_{i}-\delta_{B I}-\delta_{H I} / 2} \times \dot{\alpha},
\end{aligned}
$$

where $\overrightarrow{x_{A}}$ is the unit vector for the tangential direction at the contact point $A$, having the angle $\beta+\gamma$ with $\overrightarrow{o x_{G}}$ in Figure 7 , and $\overrightarrow{x_{B}}$ is the unit vector for the tangential direction at the contact point $A$.

The similar results are also obtained for the pure rolling in contact point $B$ in Figure 7

$$
\dot{\theta}_{b e}=\dot{\varphi}-\frac{b_{e}}{R_{e}-\delta_{B E}-\delta_{H E} / 2} \times \dot{\alpha} .
$$

Then the following result is obtained by getting the difference between (A.4) and (A.5)

$$
\begin{aligned}
\dot{\theta}_{b i}- & \dot{\theta}_{b e} \\
= & -\left(\frac{b_{i}}{R_{i}-\delta_{B I}-\delta_{H I} / 2}-\frac{b_{e}}{R_{e}+\delta_{B E}+\delta_{H E} / 2}\right) \\
& \times \dot{\alpha} .
\end{aligned}
$$

Because the time function parameters $b$ and deformations in (A.6) have little change in simulation, so the windup angle can be expressed as follows by the integration of (A.6).

$$
\begin{aligned}
\theta_{b i}- & \theta_{b e} \\
= & -\left(\frac{b_{i}}{R_{i}-\delta_{B I}-\delta_{H I} / 2}-\frac{b_{e}}{R_{e}+\delta_{B E}+\delta_{H E} / 2}\right) \\
& \times \alpha .
\end{aligned}
$$

Based on the static equilibrium equations for the sprag, the expression of the torque transmitted can be written as

$$
=\frac{n_{s} \times h_{i} \times N_{b i}}{b e /\left(R_{e}+\delta_{B E}+\delta_{H E} / 2\right)+b_{i} /\left(R_{i}-\delta_{B I}-\delta_{H I} / 2\right)} .
$$

So the torsional stiffness is obtained by the division between the torque transmitted and the windup angle.

$$
T \text { _stiffness }=\frac{C}{\theta_{b i}-\theta_{b e}} .
$$

As shown in Figure 11, the torsional stiffness considering two types of deformations has better agreement with FEM results than the torsional stiffness only considering the local deformation. Further, based on the two types of deformations, Figure 12 shows that, compared with the results in which the local deformation is calculated by Hertz theory, the torsional stiffness using the slice method has better agreement with FEM results. 


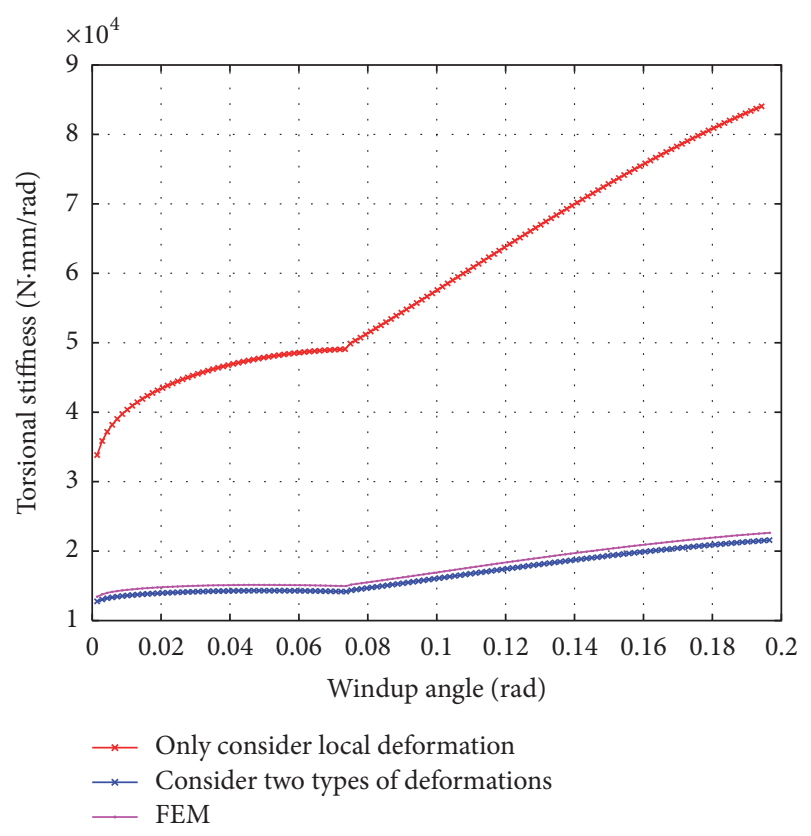

FIGURE 11: Comparison of the effects of local deformation and structural deformation.

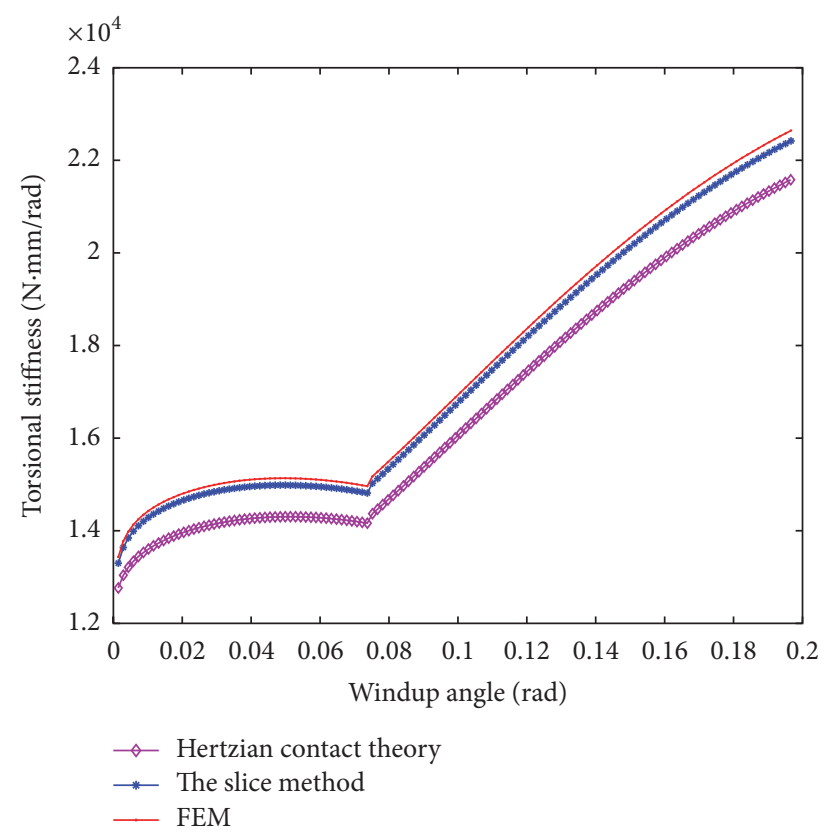

FIGURE 12: Torsional stiffness calculated by different methods.

\section{Conflicts of Interest}

The authors declare that they have no conflicts of interest.

\section{Acknowledgments}

This work was supported by the National Natural Science Foundation of China (Grant no. 51505100).

\section{References}

[1] P. Lynwander, A. G. Meyer, and S. Chachakis, "Sprag overrunning clutch," Tech. Rep., U.S Army Research and Technology Laboratory, Fort Eustis, Va, USA, 1972.

[2] J. Kish, "Helicopter free wheel unit design guide," Report, Fort Eustis, U.S Army Reaearch and Technology Laboratoty, Virginia, Va, USA, 1977.

[3] C. Chassapis and G. G. Lowen, "High speed dynamics of a press feed mechanism, part I: theory," in Proceedings of the ASME 21st Biennal Mechanisms Conference, pp. 415-437, Chicago, Ill, USA, 1990.

[4] T. Xu and G. G. Lowen, "A new damping model for nonlinear stiffness systems with variable preload displacements and constant amplitude decay ratios," Journal of Mechanical Design, vol. 116, pp. 257-263, 1994.

[5] T. Xu and G. G. Lowen, "A mathematical model of an overrunning sprag clutch," Mechanism and Machine Theory, vol. 29, no. 1, pp. 11-23, 1994.

[6] Z.-H. Liu, H.-Z. Yan, and Y.-M. Cao, "Design and analysis of logarithmic spiral type sprag one-way clutch," Journal of Central South University, vol. 22, no. 12, pp. 4597-4607, 2015.

[7] P. Vernay, Comportement dynamique en torsion et en regime transitoire d'un deHmarreur de moteur d'avion [Ph.D. thesis], l'INSA de Lyon, Villeurbanne, France, 1999.

[8] P. Vernay, G. Ferraris, A. Delbez, and P. Ouplomb, "Transient behaviour of a sprag-type over-running clutch: an experimental study," Journal of Sound and Vibration, vol. 248, no. 3, pp. 567$572,2001$.

[9] H. Ngo, R. Weisenburger, D. Knapp et al., "Condition based maintenance integration diagnostics/prognostics of the AH-64 main transmission accessory sprag clutch," in Proceedings of the 67th American Helicopter Society International Annual Forum, pp. 1532-1549, Virginia Beach, Va, USA, May 2011.

[10] G. Ochse, R. Schonen, and C. Trabing, "MBS simulation techniques to determine spatial load distributions. and torque build-up for freewheel clutches. Report," in Proceedings of the SIMPACK User Meeting, 2014.

[11] H. Fujiwara and T. Kawase, "Logarithmic profile of rollers in roller bearing and optimization of the profile," Transactions of the Japan Society of Mechanical Engineers, Series C, vol. 72, no. 9, pp. 3022-3029, 2006.

[12] R. Teutsch and B. Sauer, "An alternative slicing technique to consider pressure concentrations in non-Hertzian line contacts," Journal of Tribology, vol. 126, no. 3, pp. 436-442, 2004.

[13] G. U. Jun, Y. Hongzhi, Z. Zhibing et al., "Contract stress analysis of sprag overrunning clutch and life prediction," Modern Manufacturing Engineering, vol. 03, pp. 79-82, 2009.

[14] R. I. Leine, D. H. van Campen, A. De Kraker, and L. van Den Steen, "Stick-slip vibrations induced by alternate friction models," Nonlinear Dynamics, vol. 16, no. 1, pp. 41-54, 1998.

[15] R. I. Leine, Bifurcations in discontinuous mechnical system of filippov-type [Ph.D. thesis], Technical University of Eindboven, 1999.

[16] D. Karnopp, "Computer simulation of stick-slip friction in mechanical dynamic systems," Journal of Dynamic Systems, Measurement and Control, Transactions of the ASME, vol. 107, no. 1, pp. 100-103, 1985.

[17] K. L. Johnson and R. Cameron, "Shear behavior of elastohydrodynamic oil film at high rolling contact pressures," Proceedings of the Institution of Mechanical Engineers, vol. 182, article 307, 1967. 


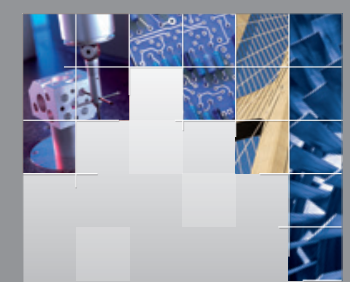

\section{Enfincering}
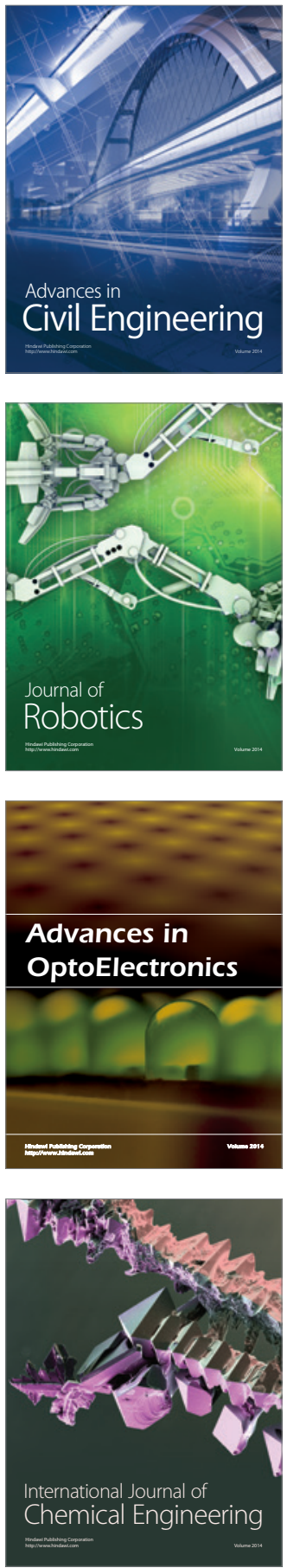

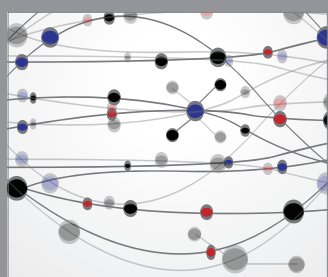

The Scientific World Journal

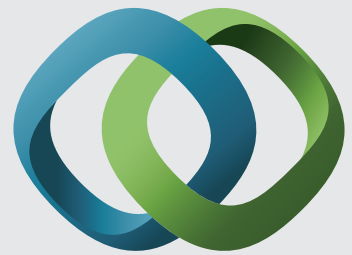

\section{Hindawi}

Submit your manuscripts at

https://www.hindawi.com
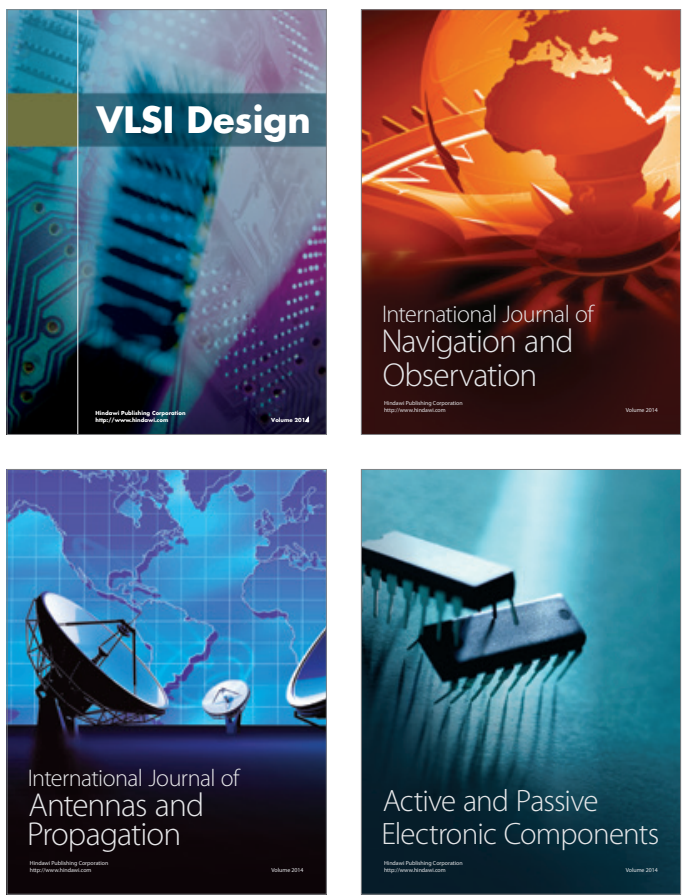
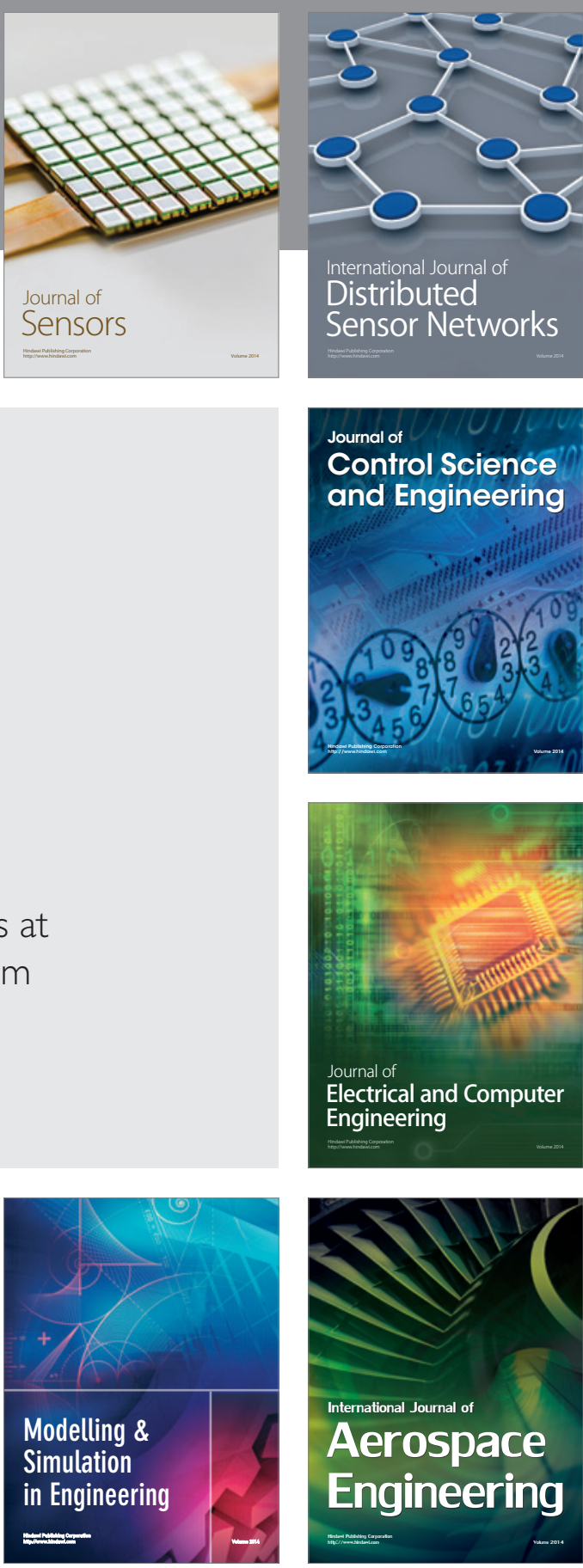

International Journal of

Distributed

Sensor Networks

$-$

Joumal of

Control Science

and Engineering
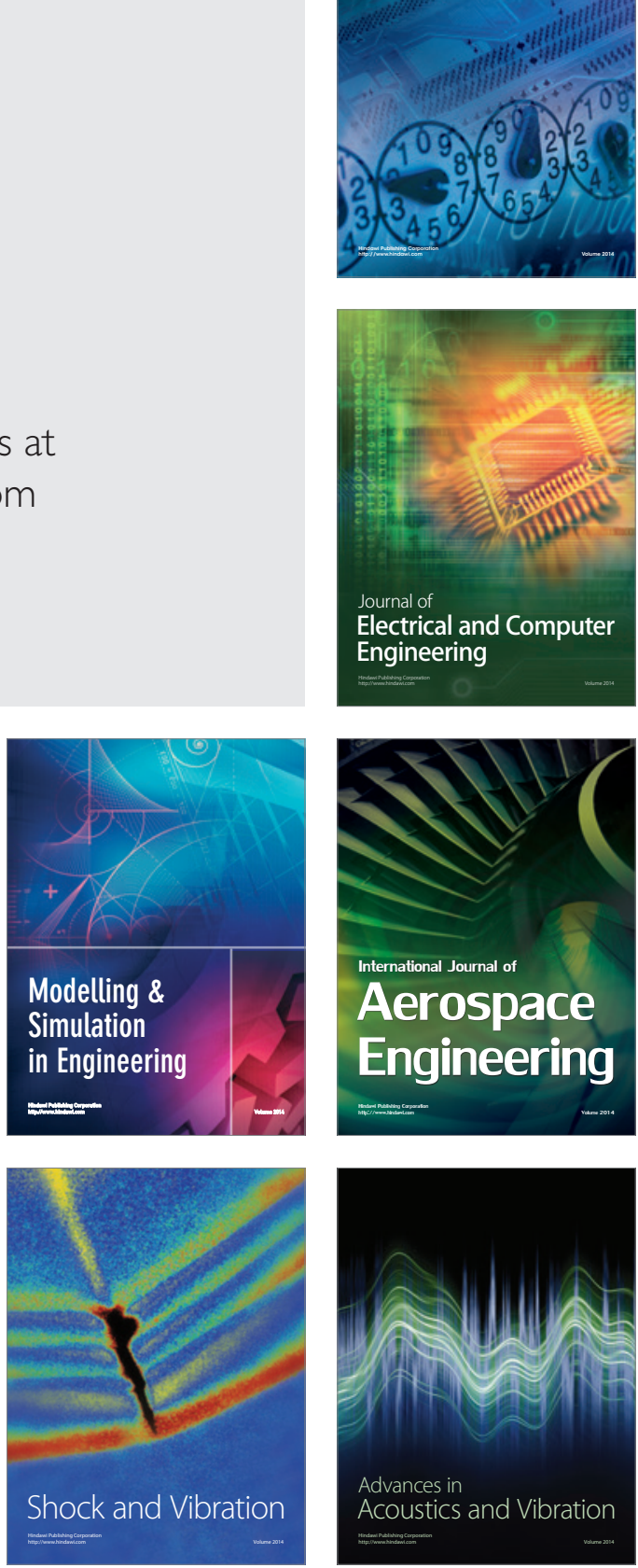